\title{
Crystal defects and their impact on ribbon growth on substrate (RGS) silicon solar cells
}

\author{
U. Hess ${ }^{\text {a,* }}$, P.Y. Pichon ${ }^{\text {b }}$, S. Seren ${ }^{\text {a }}$, A. Schönecker ${ }^{\text {b }}$, G. Hahn ${ }^{\text {a }}$ \\ a University of Konstanz, Department of Physics, P.O. Box X916, 78457 Konstanz, Germany \\ ${ }^{\mathrm{b}}$ RGS Development B.V., P.O. Box 40,1724ZG Oudkarspel, The Netherlands
}

\section{A R T I C L E I N F O}

Keywords:

Ribbon growth on substrate

Multicrystalline silicon

Solar cells

Crystal defects

\begin{abstract}
A B S T R A C T
Ribbon Growth on Substrate (RGS) silicon wafers are casted directly from the silicon melt onto reusable substrates. Material losses by wafer sawing are omitted and high production speeds can be achieved. However, multicrystalline RGS silicon as it is produced today incorporates high densities of crystal defects and impurities limiting the efficiency of the corresponding solar cells. The local impact of crystal defects on material quality is estimated via models developed by Donolato and Micard et al.. By theoretically negating the impact of grain boundaries and dislocations, charge carrier diffusion lengths are still limited to values $<100 \mu \mathrm{m}$. In addition to crystal defects which are common in other multicrystalline silicon materials, we found current collecting structures within grain boundaries. These structures can be associated with carbon and oxygen precipitation and are the cause for shunting phenomena. We conclude that high impurity concentrations are the dominant factor for limiting the performance of RGS silicon solar cells.
\end{abstract}

\section{Introduction}

By decoupling of wafer pulling and crystallization direction, Ribbon Growth on Substrate (RGS) [1] silicon wafers can be cast with a production speed in the order of one wafer per second. Kerf less wafering can lead to a substantial cost reduction for solar cells [2]. The RGS wafers presented in this paper are produced by RGS Development on a laboratory scale R\&D machine located at ECN (Netherlands). Using a screen printing based cell process, the highest efficiency reported is $\eta=13.1 \%$ [3] (cell size: $5 \times 5 \mathrm{~cm}^{2}$ ). One of the most influential factors on cell performance is the low shunt resistance, with strongly fluctuating values in the range of $1001000 \Omega \mathrm{cm}^{2}$. For the further development of the RGS technol ogy, understanding the cause of the varying shunt resistance and to overcome this limitation would result in a strong improvement of the quality of the wafers.

RGS material quality is decreased by several crystal defects. Grain sizes are in the order of $50500 \mu \mathrm{m}$. The dislocation densities are in the range of $10^{5} 10^{7} / \mathrm{cm}^{2}$. For the microscopy image in Fig. 1, a cross section of a RGS wafer was mechanically polished and treated with Secco etch [4]. The columnar grain structure as well as etch pits (small black dots) is observable, revealing the location of dislocations. Due to the casting method,

\footnotetext{
* Corresponding author. Tel.: +49 7531 882060; fax: +49 7531883895

E-mail address: uwe.hess@uni-konstanz.de (U. Hess).
}

defect rich layers (thickness $\sim 20 \mu \mathrm{m}$ ) are present on both wafer surfaces.

Impurity concentrations as measured by Fourier transform infra red spectroscopy (FTIR) for substitutional carbon $\left[\mathrm{C}_{\mathrm{s}}\right]$ are above $10^{18} / \mathrm{cm}^{3}$ and for interstitial oxygen $\left[\mathrm{O}_{\mathrm{i}}\right]>10^{17} / \mathrm{cm}^{3}$. The actual concentrations are higher, since e.g. precipitates are not detected. The minority charge carrier lifetime in $\mathrm{p}$ type boron doped ( $3 \Omega \mathrm{cm}$ ) as grown wafers is in the order of $\sim 1 \mu \mathrm{s}$. After cell processing steps like phosphorus gettering (emitter diffusion) and hydrogenation ( $\mathrm{SiN}_{\mathrm{x}}: \mathrm{H}$ deposition and firing), the life time increases to values of about $510 \mu \mathrm{s}$ in RGS wafers. The diffusion length of minority charge carriers is in the range of $6090 \mu \mathrm{m}$, as determined from internal quantum efficiency (IQE) measurements.

To evaluate the impact of different crystal defects on the material quality, several measurement methods were used and the results correlated. Light Beam Induced Current (LBIC), Lock In Thermography (LIT) and Photoluminescence (PL) were chosen as spatially resolved measurement methods for the characterization on cell level. Subsequently, the characterized solar cells were etched and the surfaces were mechanically polished (prior to the cell process, the defect rich surfaces were already removed by acidic etching). Scanning Electron Microscopy (SEM), optical as well as infrared microscopy were used to investigate crystal defects. To detect the concentrations of metallic impurities high resolution Inductively Coupled Plasma Mass Spectroscopy (ICP MS) measurements were carried out. 


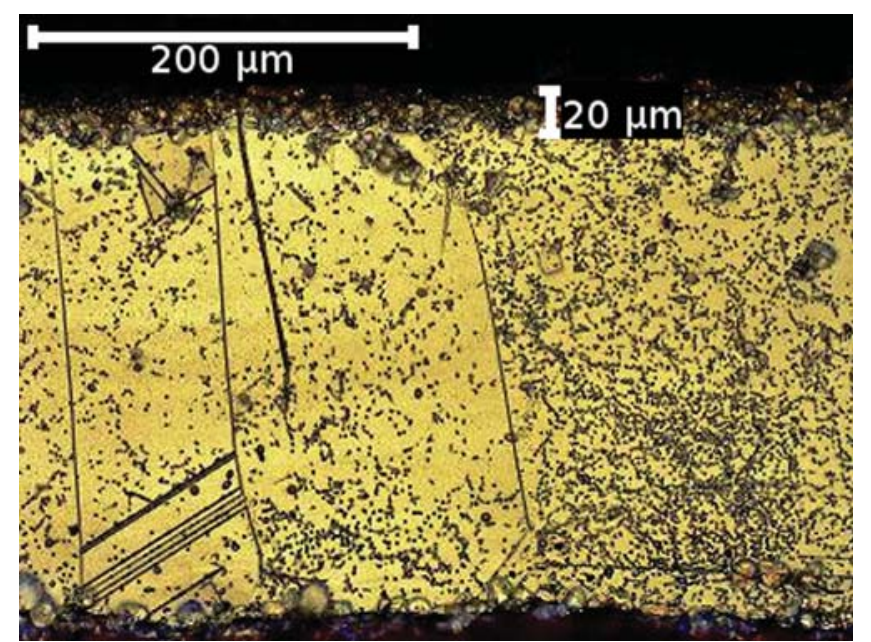

Fig. 1. Optical microscopy image of a polished cross-section of a RGS wafer treated with Secco etch.

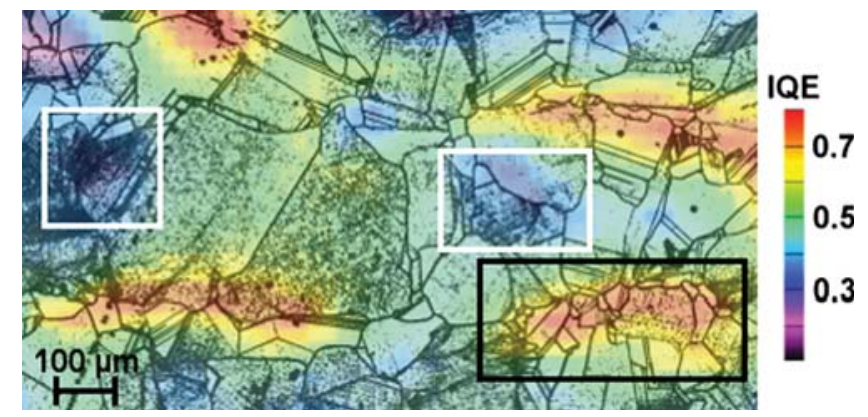

Fig. 2. Overlay of a LBIC scan ( $\lambda 980 \mathrm{~nm}$, resolution: $25 \mu \mathrm{m}$ ) with the optical microscopy image of the corresponding polished and Secco-etched wafer. Defective regions (white rectangles) and a feature that is associated with a current collecting structure (black rectangle, structure has measured IQE values of $>0.7$ ) are highlighted.

\section{Results}

Fig. 2 shows a section of the overlay of a LBIC scan $(980 \mathrm{~nm}$ wavelength) and the optical microscopy image of the correspond ing polished and Secco etch treated wafer. The accurateness of the overlay is in the order of the LBIC pixel width $(25 \mu \mathrm{m})$. Like the highlighting in Fig. 2 indicates, the IQE is locally and distinctively varying from the average value. The following subsections are dealing with the different features of these overlay images.

\subsection{Grain boundaries}

As can be observed in Fig. 2, grain boundaries can show different behaviors in terms of short circuit current or IQE. Several models were proposed to theoretically describe the recombinative behavior of grain boundaries [5]. However, these would require detailed knowledge of trapping and capture cross sections of charge carriers at the grain boundary. Due to the grain size and distribution, corresponding measurements would be problematic.

To circumvent this problem, a method developed by Micard et al. [6] is used. This method uses a high resolution LBIC line scan over grain boundaries. By theoretical considerations, the recombi native behavior of a grain boundary can be described by an effective surface recombination velocity $\mathrm{S}_{\mathrm{GB}}$. A defective region including several grain boundaries, whereby the influence of an isolated one cannot be distinguished, can also be described by an effective $\mathrm{S}_{\mathrm{GB}}$ by the same model. For the evaluation on RGS samples, an exploitable drop in short circuit current is only observable in defective regions. The majority (90\%) of all investi gated defective regions show varying $\mathrm{S}_{\mathrm{GB}}$ in the range of 1000 $50,000 \mathrm{~cm} / \mathrm{s}$. Single grain boundaries do rarely show such a distinctive behavior. Due to measurement noise, it can only be stated that the effective surface recombination velocities of most isolated grain boundaries are in the range below $\sim 1000 \mathrm{~cm} / \mathrm{s}$. In grains with a size of several diffusion lengths and not being located in the vicinity of defective regions, recombination at grain boundaries has no dominant influence on material quality. This circumstance is also used in the next subsection for evaluation of the impact of dislocations.

\subsection{Dislocations}

To estimate the recombinative behavior of dislocations in RGS, a model developed by Donolato is used [7]. This theoretical consideration allows a correlation of the average normalized line recombination velocity $\Gamma$ of dislocations, the charge carrier diffu sion length and the so called background diffusion length, which corresponds to the diffusion length if no dislocation would be present. On multicrystalline material, this model was successfully applied by Rinio et al. [8]. To apply this model to RGS several assumptions have to be made.

Due to the ratio of diffusion length $(<100 \mu \mathrm{m})$ to cell thickness (220 $250 \mu \mathrm{m}$ ), the cell can be approximately treated as a semi infinite semiconductor. Since no preferred orientation of disloca tions could be observed, a random orientation with an average angle of $45^{\circ}$ between dislocations and surface is assumed. Since the influence of grain boundaries on the evaluation has to be minimized, determination of dislocation density and local diffu sion length is narrowed to regions in the center of grains with a size of at least five times the diffusion length and with no defective region in the vicinity. Due to these criteria, only 150 measure ment points could be obtained from the investigated cell.

The dislocation density is determined by counting of etch pits on the Secco etched wafer and the local diffusion length is determined by LBIC measurements with three different wave lengths $(\lambda=833,910,980 \mathrm{~nm})$ and a subsequent fit according to Basore [9]. Fig. 3 shows the obtained results.

Concerning the error margins, etch pit density determination and density variability within a grain lead to an uncertainty in the order of $1020 \%$. Above a density of $10^{7} / \mathrm{cm}^{2}$ the determination was increasingly unreliable. Due to the method of determining the local diffusion length, an error margin of about $5 \mu \mathrm{m}$ has to be assumed.

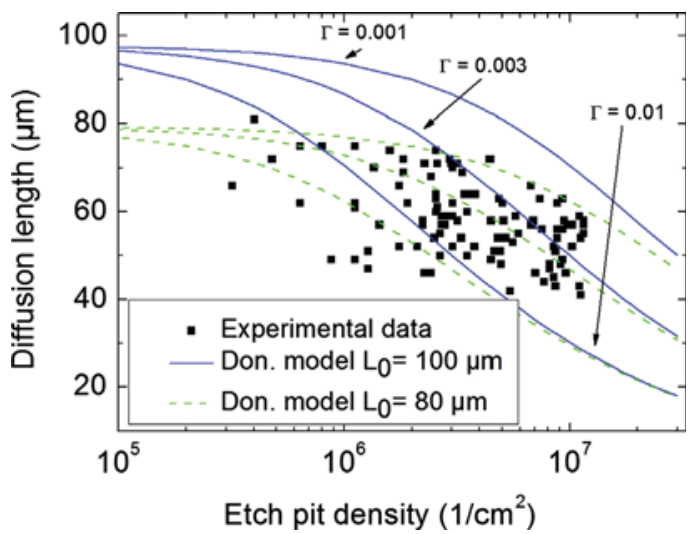

Fig. 3. The dashed green and the solid blue curves serve as guide to the eye for the theoretical Donolato model with two different background diffusion lengths $L_{0}$ and three different line recombination velocity values, each. The experimental data is gained by local determination of etch pit density and diffusion length. (For interpretation of the references to color in this figure caption, the reader is referred to the web version of this article.) 
Due to the uncertainties of the theoretical assumptions and the experimental procedures in combination with the scattering of the experimental data, a statement about the average line recombina tion velocity cannot be given. The blue and green curves in Fig. 3 are drawn as guide to the eye. However, information about the background diffusion length can be confidently gained. By theore tically negating the impact of grain boundaries and dislocations, the diffusion length is still limited to values $<100 \mu \mathrm{m}$. Thus, the dominant factor for RGS bulk material quality is likely to be found in impurities and corresponding precipitation.

\subsection{Current collecting structures}

In Fig. 2, unusually high IQE values can be found around certain grain boundaries. Using infrared microscopy, light scattering dark

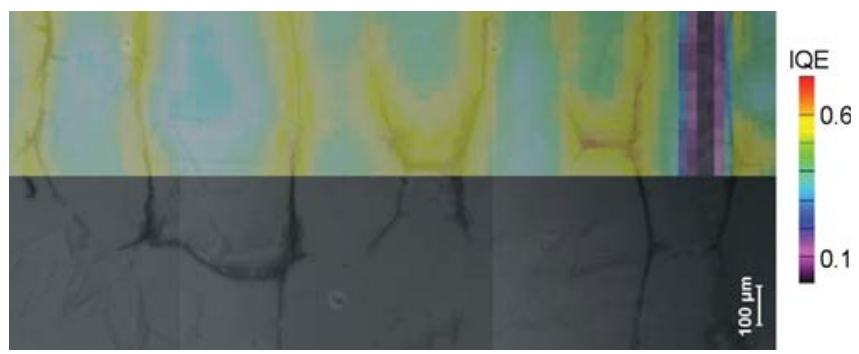

Fig. 4. Top surface scan showing an infrared microscopy image (gray image) overlaid with the corresponding local IQE map (color image) obtained by LBIC ( $\lambda 980 \mathrm{~nm}$, resolution: $25 \mu \mathrm{m}$ ). (For interpretation of the references to color in this figure caption, the reader is referred to the web version of this article.) structures can be found in grain boundaries within RGS wafers. Investigations showed voids as well as carbon and oxygen based precipitates (shown in Section 2.4) in these grain boundaries [10]. Due to their ability to scatter light they are also referred as "dark grain boundaries". The locations of these dark structures coincide with the unusual high IQE values, as is shown in Fig. 4.

Fig. 5 shows the LBIC, iLIT (illuminated Lock In Thermography) and PL measurement images of the same cell area. The structures are too small and too dense to allow the observation of any isolated effects with Lock In Thermography. However, the PL image shows local shadowing, which can be associated with recombination and/or shunting.

To investigate the electrical behavior of these structures, the cell was cut and the cross section mechanically polished. An Electron Beam Induced Current (EBIC) measurement was con ducted in a SEM setup. The electrons are injected into the plane of the cross section. At the locations of the dark structures a strong signal can be found. In case of the structure shown in Fig. 6, the dark grain boundary shows a current collecting behavior starting from the front surface (left image) through the complete silicon bulk to the Al back contact (right image).

Up to now, all RGS cells characterized by LBIC in this study show locations with such unusually high IQE values. However, the density is not constant. For the corresponding cell of the measurements shown in Fig. 5, for $\sim 75 \%$ of the cell area the areal density of these structures density is in the range of $100200 / \mathrm{cm}^{2}$. In the remaining $25 \%$ of the cell area, the structure density is considerably lower with densities in the range of $\sim 10 / \mathrm{cm}^{2}$. To investigate the impact of the structure density on the solar cell parameters, the sample was cut in small cells $\left(1 \times 1 \mathrm{~cm}^{2}\right)$ and re measured. The most significant difference of the small cells can be found in the shunt resistance. For cells with a high
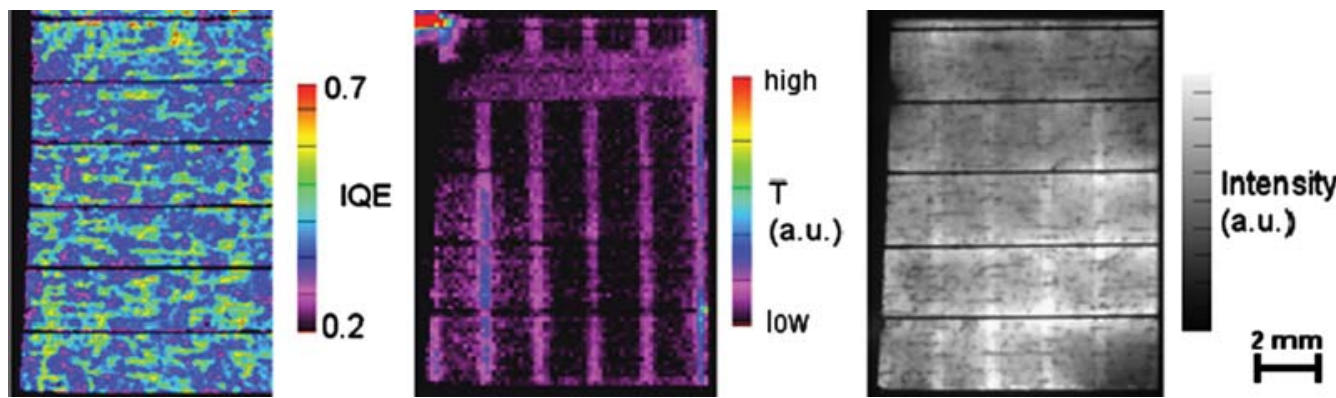

Fig. 5. LBIC at $980 \mathrm{~nm}$ (left), iLIT (middle) and PL (right) measurements of the same section of a RGS cell. The RGS cell shown here does not have a full area Al back contact but a grid (observable in the iLIT and the PL images as vertical lines) to reduce the probability of contacting a shunting structure.
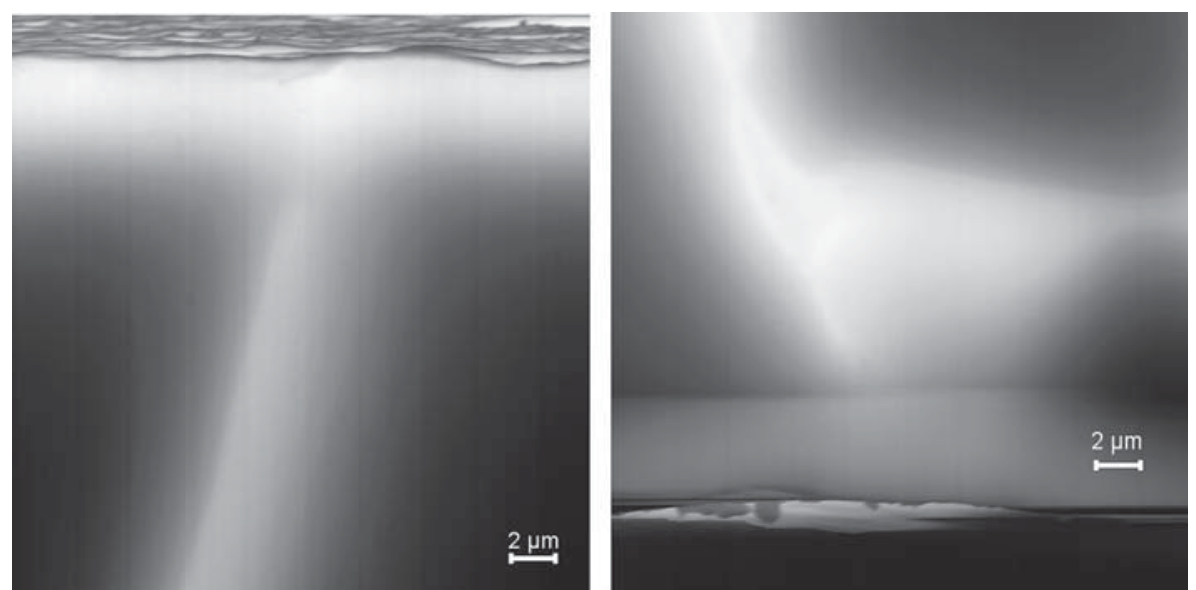

Fig. 6. EBIC scan of a cross-section of a RGS cell. A current collecting structure at the location of a dark grain boundary can be observed. The structure reaches from the emitter (top of left image) completely through the silicon bulk to the Al back contact (bottom of right image). 


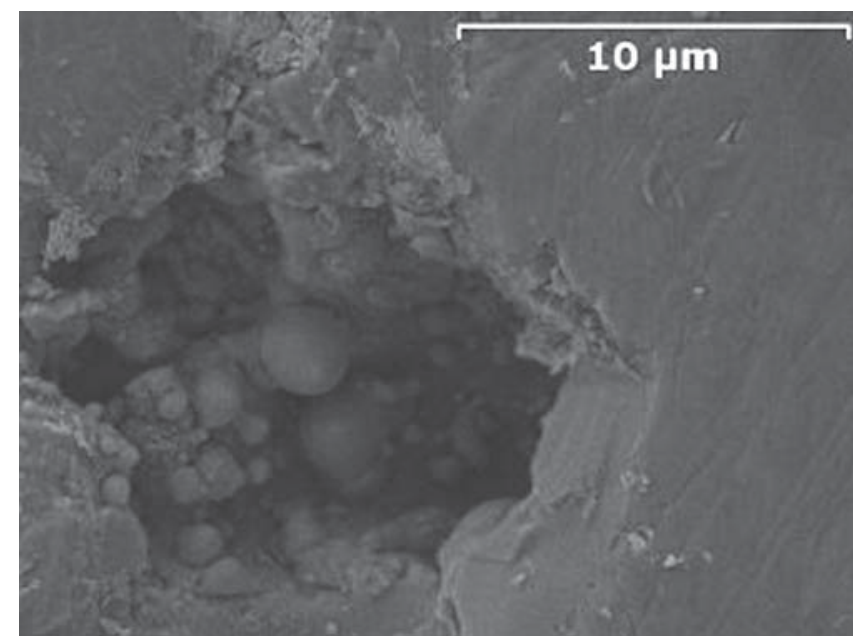

Fig. 7. SEM image at the location of a dark grain boundary. Voids as well as carbon and oxygen precipitates (via EDX) can be observed.

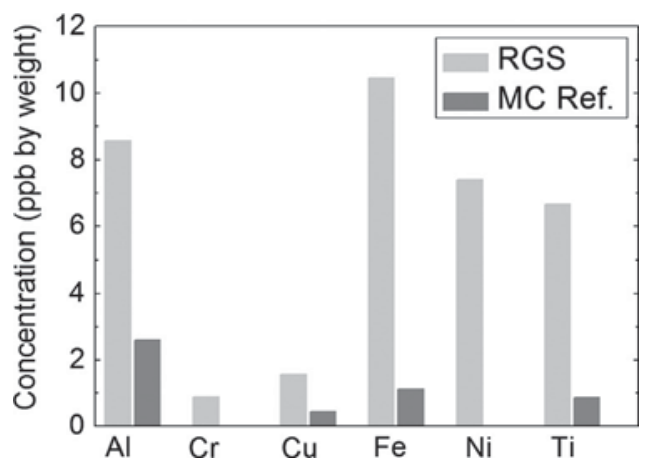

Fig. 8. Metallic impurity concentrations of a typical RGS wafer produced in the laboratory machine in comparison to commercial multicrystalline silicon wafers. Measurements were done by high resolution ICP MS. The values for $\mathrm{Cr}$ and Ni for the multicrystalline references were below the detection limit ( $<0.1 \mathrm{ppb})$.

density of dark grain boundaries, the shunt resistance is in the range of $100300 \Omega \mathrm{cm}^{2}$. For cells with a low structure density, the shunt resistance is in the range of $\sim 1500 \Omega \mathrm{cm}^{2}$.

\subsection{Impurities}

Impurity concentrations at the location of dark grain boundaries were determined by Energy Dispersive X ray spectroscopy (EDX). For the SEM image in Fig. 7, a wafer cross section was mechanically polished. Small voids with oxygen and carbon precipitates can be observed. EDX revealed silicon with an oxygen content of approxi mately $5 \%$ and a carbon content of approximately $10 \%$ (mass percent). Due to the size of the measurement spot of the EDX setup compared to the size of the observed structures, these values must be considered as lower limits for the real concentrations.

Besides FTIR measurements for average $\left[\mathrm{C}_{\mathrm{s}}\right]$ and $\left[\mathrm{O}_{\mathrm{i}}\right]$ values, ICP MS measurements for metallic impurities were carried out. In Fig. 8, a comparison of impurity concentrations in RGS compared to multicrystalline block cast silicon is shown. In combination with the carbon and oxygen content, this is the most dominant cause for the low diffusion lengths in RGS silicon.

\section{Discussion}

The varying effective recombination velocities at grain bound aries (and defective regions) lead to the conclusion, that this distortion of the crystal lattice is not the dominant factor for increased recombination. Judging from the impurity concentra tions and that current collecting structures can be associated with carbon and oxygen precipitation, it is most likely that impurities are the decisive factor for a grain boundary being nearly inactive, acting as defective region or as current collecting structure.

The estimation of the impact of dislocations on the material quality suffers from uncertainties in measurement and local variations. The average normalized line recombination velocity $\Gamma$ of the dislocations cannot be evaluated. However, the background diffusion length can be estimated. It can be stated that even by theoretically negating the effect of dislocations and grain bound aries, the material quality in terms of the diffusion length is low compared to other ribbon (e.g. Edge defined Film fed Growth, EFG) or blockcast multicrystalline materials. A possible cause for this behavior could be the relatively high metal impurity concen trations in this material.

The current collecting structures within grain boundaries seem to be the main factor for the generally low shunt resistances of RGS solar cells. For older RGS material with quality suffering from higher concentrations of oxygen $\left(\left[\mathrm{O}_{\mathrm{i}}\right]>10^{18} / \mathrm{cm}^{3}\right)$, carrier collect ing channels have also been found [11]. However, these channels were much smaller and could be associated with dislocations. In today's material from the laboratory RGS machine, the character ization results indicate a correlation between $\mathrm{SiC}$ formation and carrier collecting structures. However, it should be mentioned that segregating metal impurities are likely to be found in the same area where SiC formation can be observed.

\section{Conclusion}

The dominant factor for the recombination in RGS silicon wafers is the local impurity concentration. The silicon bulk is theoretically limited to diffusion lengths $<100 \mu \mathrm{m}$. Agglomeration of impurities at crystal defects decrease the material quality further. Also, precipitation at grain boundaries can lead to current collecting structures which strongly decrease the shunt resistance of the solar cells.

These detailed wafer characterization results are an important contribution in the development of the future RGS material improvement program. The first focus in developing and upgrad ing the RGS casting process is on contamination control both on reducing metal contamination as on reducing the carbon concen tration. Other crystal imperfections such as dislocations or the small grain sizes are not yet the limiting factor for the material quality.

\section{Acknowledgments}

Part of this work was funded by the German Federal Ministry for Environment, Nature Conservation and Nuclear Safety in the OP RGS (0325056) project. The supply of RGS wafers by RGS Development B.V. is gratefully acknowledged. The content of this publication is the responsibility of the authors.

\section{References}

[1] H. Lange, I.A. Schwirtlich, Ribbon Growth on Substrate (RGS) - A new approach to high speed growth of silicon ribbons for photovoltaics, Journal of Crystal Growth 104 (1990) 108.

[2] G. Hahn, S. Seren, M. Kaes, A. Schönecker, J.P. Kalejs, C. Dubé, A. Grenko, C Belouet, Review on ribbon silicon techniques for cost reduction in PV, in: Proc. 4th WC-PEC (2006) 972.

[3] S. Seren, M. Kaes, G. Hahn, A. Gutjahr, A.R. Burgers, A. Schönecker, Efficiency potential of RGS silicon from current R\&D production, in: Proc. 22nd EC-PVSEC (2007) 854. 
[4] D.G. Schimmel, A comparison of chemical etches for revealing $\langle 100\rangle$ silicon crystal defects, Journal of The Electrochemical Society 123 (1976) 734

[5] S.A. Edminston, G. Heiser, A.B. Sproul, M.A. Green, Improved modeling of grain boundary recombination in bulk and pn junction regions of polycrystalline silicon solar cells, Journal of Applied Physics 80 (1996) 6783.

[6] G. Micard, G. Hahn, A. Zuschlag, S. Seren, B. Terheiden, Quantitative evaluation of grain boundary activity in multicrystalline semiconductors by light beam induced current: an advanced model, Journal of Applied Physics 108 (2010) 1.

[7] C. Donolato, Modeling the effect of dislocations on the minority carrier diffusion length of a semiconductor, Journal of Applied Physics 84 (1998) 2656.
[8] M. Rinio, S. Peters, M. Werner, A. Lawerenz, H.J. Möller, Measurement of the normalized recombination strength of dislocations in multicrystalline silicon solar cells, Solid State Phenomena 82 (2002) 701.

[9] P.A. Basore. Extended spectral analysis of internal quantum efficiency, in: Proc. 23th IEEE-PVSC (1993) 147.

[10] U. Hess, S. Joos, S. Seren, G. Hahn, P.-Y. Pichon, A. Schönecker, T. Weber, Infrared microscopy investigation of the crystal structure of ribbon growth on substrate (RGS) solar sells, in: Proc. 24th EC-PVSEC (2009) 2138.

[11] G. Hahn, D. Sontag, C. Haessler, Current collecting channels in RGS silicon solar cells - are they useful? Solar Energy Materials and Solar Cells 72 (2002) 453. 